\title{
Neural Mechanisms of Social Reorientation across Adolescence
}

\author{
Wouter van den Bos \\ Department of Psychology, Stanford University, Stanford, California 94305 \\ Review of Pfeifer et al.
}

Adolescence is characterized by a unique set of physical, hormonal, and neural changes. In addition to changes in the body, adolescence is marked by great changes in social behavior. During this period in humans, there is an increase in interest in, and time spent with, peers and romantic partners, and adolescents establish an individual identity. This process of social reorientation is one of the most salient behavioral changes of adolescence (Nelson et al., 2005). It is hypothesized that changes in the social contexts that afford social learning, in combination with a specific set of changes in cognitive and affective systems, facilitate adolescent social reorientation (Crone and Dahl, 2012). More specifically, developmental theories suggest that changes in social behavior are related to increased perspectivetaking and self-regulation skills (Blakemore and Robbins, 2012) and to increased appetitive motivational tendencies in the realm of social goals and rewards (Forbes and Dahl, 2010). Animal models suggest that the pubertal rise in reproductive hormones is an important factor in the activation of the social motivational changes (Spear, 2010).

A major challenge in developmental neuroscience is understanding how these

Received June 24, 2013; revised July 16, 2013; accepted July 21, 2013.

This work was supported by Netherlands Organization for Scientific Research (NW0) Rubicon Postdoctoral Fellowship 446-11-012. I thank Ron Dahl for helpful comments and suggestions.

Correspondence should be addressed to Wouter van den Bos, Department of Psychology, Stanford University, 450 Serra Mall, Stanford, CA 94305. E-mail:wvdbos@stanford.edu.

DOI:10.1523/JNEUROSCI.2667-13.2013

Copyright $\odot 2013$ the authors $\quad 0270-6474 / 13 / 3313581-02 \$ 15.00 / 0$ different hormonal, neural, and contextual changes interact in ways that lead to successful social reorientation. In the past decade, several fMRI studies on adolescent social development have been conducted (Blakemore and Robbins, 2012). Although these studies have increased our understanding of the relation between brain development and social reorientation, most of these studies have been cross-sectional, focusing on age-related changes, and ignoring pubertal development. As a result, the respective roles of puberty and age in adolescent social reorientation remain unclear.

In their recent article in The Journal of Neuroscience, Pfeifer et al. (2013) were among the first to investigate within-subject neural changes related to age and puberty by applying a longitudinal fMRI approach. In this study, 27 youths were scanned while reporting whether short phrases concerning social and academic attributes described themselves or a highly familiar fictional character, Harry Potter (before the scanning sessions it was ensured that all participants were sufficiently familiar with Harry Potter). Sample phrases included: "I am popular," "I wish I had more friends," "I like to read just for fun," and "Writing is so boring." This design allowed the investigators to study neural responses associated with self versus other judgments as well as social versus nonsocial judgment.

The average age of the participants was $10.1(\mathrm{SD}=0.35)$ at the first scan (T1), and $13.1(\mathrm{SD}=0.33)$ during the second scan (T2). To get a measure of pubertal development, participants self-reported about observed changes in visible secondary sex characteristics (e.g., pubic hair, skin problems, genital development) using the Pubertal Development Scale (PDS). As expected, the authors found a significant increase in pubertal development from $\mathrm{T} 1$ to $\mathrm{T} 2$.

The longitudinal design allowed investigation of both stability and change in neural patterns, in relation to age and puberty. First, the authors established the main effect of target (self vs fictional other). Responses in medial prefrontal cortex (mPFC) and ventral striatum (VS) were greater during self evaluations, whereas responses in the medial posterior parietal cortex (mPPC), lateral prefrontal cortex (IPFC), and temporoparietal junction (TPJ) were greater during evaluations of an imaginary other. Furthermore, conjunction analyses revealed that activation patterns in MPFC, VS, and $\mathrm{mPPC}$ were stable over time. More interestingly, the authors also identified an area in the ventral $\mathrm{mPFC}$ (vmPFC) that showed an age-related increase in difference in activation during evaluations of self versus fictional other. Post hoc ROI analyses were performed to further investigate the developmental changes in the vmPFC. These analyses revealed two interesting findings: (1) that level of pubertal development, even when controlling for age, was significantly associated with the increased difference in vmPFC activation during evaluations of self versus fictional other; and (2) that this effect was only significant for statements about social interactions and not statements about academic performance. These results are 
consistent with several cross-sectional studies of social reorientation that have shown that there is increased activation in the $\mathrm{mPFC}$ related to self-relevant processing in early adolescence (Blakemore and Robbins, 2012). Importantly, these results extend earlier findings by showing that these early increases in $\mathrm{mPFC}$ engagement are related to pubertal development independent of age. Furthermore, the study reports that TPJ activity, associated with evaluation of others, was not related to pubertal development. In contrast, numerous studies have shown that the TPJ is associated with age-related changes in adolescent social behavior (Blakemore and Robbins, 2012). Taken together, these results suggest that there are dissociable developmental processes that contribute to adolescent social reorientation, some more related to pubertal development (vmPFC) and others more related to age (TPJ). Although this pattern of results deepens our knowledge of the mechanisms underlying adolescent social reorientation, it also raises several interesting questions.

First, it raises the question: which specific mechanisms of pubertal development contribute to which specific changes in brain function? Puberty as measured with the PDS, which focuses on physical changes, is only a proxy for a whole series of developmental processes. It is well known that the gonadal hormones, testosterone and estradiol, play a role in the development of secondary sexual characteristics, but these characteristics are also influenced by the adrenal androgens dehydroepiandrosterone (DHEA) and DHEA sulfate. Furthermore, there is evidence that other neuroendocrine systems undergo developmental changes at puberty, such as the regulation of growth hormones, the hypothalamic-pituitaryadrenal axis, oxytocin, and vasopressin (Peper and Dahl, 2013). The development of many neuroendocrine systems is correlated with measures of physical development, but they are also likely to affect different neural systems. This raises the questions: how do changes in these particular neuroendocrine systems affect specific neural systems and at which points in development? Furthermore, these hormonal changes may have different impact in boys versus girls. This is nicely illustrated by a recent study by Op de Macks and colleagues (2011) that showed that stronger activity in the ventral striatum after high-risk gambles is correlated with higher testosterone levels in boys and higher estradiol levels in girls, although the effect of estradiol was weaker than that of testosterone. Interestingly, both estradiol and testosterone have been associated with individual differences in social behavior. Therefore, these hormones are likely contributors to adolescent social reorientation. However, another likely candidate is the oxytocin-vasopressin system. Studies in humans and animals have shown that this system is central to social behavior and influences social cognitive processes and, importantly, shows great changes during early puberty (Spear, 2010). Future studies using more detailed measurements of the different neuroendocrine systems are needed to tease this apart.

Second, the study by Pfeifer et al. (2013) shows an increase in $\mathrm{mPFC}$ activity during self-evaluations between 10- and 13-yearsold, whereas their earlier cross-sectional study (Pfeifer et al., 2007) showed a decline in mPFC activity during self-evaluations between 10-year-olds versus young adults. Given that the mPFC area in the crosssectional study was slightly more dorsal, the authors suggest these might be functionally different mPFC areas, but it could also be that this indicates a nonlinear developmental trajectory within the $\mathrm{mPFC}$ area; the often-reported increase in activation in early adolescence followed by a decrease in later adolescence. The latter interpretation is in line with a recent study on the adolescent development of self-consciousness (Somerville et al., 2013). In this study, the authors directly tested for three patterns of agerelated changes in brain activity: linear, adolescent-emergent, and adolescentspecific. Their results indicate that the developmental pattern in the $\mathrm{mPFC}$ related to self-consciousness is consistent with both adolescent-emergent and adolescent-specific trends. Unfortunately, Somerville et al.'s (2013) study did not include measures of pubertal development. Still, one possibility that is consistent with these findings and Pfeifer et al. (2013) is that the early surge of pubertal hormones causes a temporary increase in self-evaluations, which is reflected by increases in relative vmPFC activity during self-evaluation compared with other evaluation. Then, at a later stage of adolescence, there is a reduction in self-evaluation and a decrease in relative vmPFC activity during self-evaluation compared with other evaluation. Adding additional time points to the longitudinal study would provide insight about these questions. An added advantage of having more time points is that it would allow investigation of interactions between age and pubertal development. It is possible that earlier onset of puberty may have profound impact on the trajectory of social development across adolescence. For example, there is evidence that earlier onset of puberty is related to increased levels of risky and antisocial behavior (Downing and Bellis, 2009).

Given the multiplicity of simultaneous developmental changes, some related to age and others to puberty, adolescence is an interesting but very challenging developmental period to study. The study by Pfeifer et al. (2013) shows the importance of a longitudinal design and the use of a tight age range for disentangling age- and puberty-related developmental changes. Future longitudinal studies could further our understanding of the mechanisms of adolescent social reorientation by including multiple measures of pubertal development.

\section{References}

Blakemore SJ, Robbins TW (2012) Decisionmaking in the adolescent brain. Nat Neurosci 15:1184-1191. CrossRef Medline

Crone EA, Dahl RE (2012) Understanding adolescence as a period of social-affective engagement and goal flexibility. Nat Rev Neurosci 13:636-650. CrossRef Medline

Downing J, Bellis MA (2009) Early pubertal onset and its relationship with sexual risk taking, substance use and anti-social behaviour: a preliminary cross-sectional study. BMC Public Health 9:446. CrossRef Medline

Forbes EE, Dahl RE (2010) Pubertal development and behavior: hormonal activation of social and motivational tendencies. Brain Cogn 72:66-72. CrossRef Medline

Nelson EE, Leibenluft E, McClure EB, Pine DS (2005) The social re-orientation of adolescence: a neuroscience perspective on the process and its relation to psychopathology. Psychol Med 35:163-174. CrossRef Medline

Op de Macks ZA, Gunther Moor B, Overgaauw S, Güroğlu B, Dahl RE, Crone EA (2011) Testosterone levels correspond with increased ventral striatum activation in response to monetary rewards in adolescents. Dev Cogn Neurosci 1:506-516. CrossRef Medline

Peper JS, Dahl RE (2013) Surging hormones: brain-behavior interactions during puberty. Curr Dir Psychol Sci. 22:134-139. CrossRef

Pfeifer JH, Lieberman MD, Dapretto M (2007) I know you are but what am I: neural bases of self-and social knowledge retrieval in children and adults. J Cogn Neurosci 19:1323-1337. CrossRef Medline

Pfeifer JH, Kahn LE, Merchant JS, Peake SJ, Veroude K, Masten CL, Lieberman MD, Mazziotta JC, Dapretto M (2013) Longitudinal change in the neural bases of adolescent social self-evaluations: effects of age and pubertal development. J Neurosci 33:7415-7419. CrossRef Medline

Somerville LH (2013) The teenage brain: sensitivity to social evaluation. Curr Dir Psychol Sci 22:129-133.

Spear L (2010) The behavioral neuroscience of adolescence. New York: Norton. 